\title{
The Dynamic COVID-Zero Strategy in China
}

\author{
Jue Liu' ${ }^{1}$ Min Liu ${ }^{1, *}$; Wannian Liang ${ }^{2,3, *}$
}

Since its outbreak in late 2019, coronavirus disease 2019 (COVID-19) has remained a global pandemic for nearly two years, which poses a huge test on the resilience of global public health system (1). After experiencing the large-scale epidemic in February 2020, China has entered a normalization stage of prevention and control since May 2020 (2). In response to the spread of the highly transmissible Delta variant, China adopted a new strategy called "Dynamic COVID-zero" from August 2021. This strategy is a summary of China's experience in dealing with the spread of the Delta variant, considering how to control the epidemic at a higher level, at a lower cost, and in a shorter time (3). The most important purpose is to minimize the impact of the epidemic on the economy, society, production, and people's normal lives, and to balance the prevention and control of this disease with socioeconomic stability. For instance, multiple outbreaks that occurred in Beijing were controlled in 2 maximum incubation periods (within 28 days) by this strategy (Figure 1).

The "Dynamic COVID-zero" strategy is a transitional strategy to be adopted after a successful containment strategy, when the population immunity barrier is not yet established in the face of continued risk of foreign importation and high transmission of variants. This is different from the traditional containment and mitigation strategies (4). The core is to take effective and comprehensive measures to deal with localized COVID-19 cases precisely, to quickly cut off the transmission chain, and to end the epidemic in a timely manner (to "find one, end one"). In other words, China took precise prevention and control measures to quickly find, control, and cure infected people in each cluster outbreak within a specific geographic region to avoid affecting social and economic development in other regions, so as to achieve the maximum effect at the lowest cost. When there is a local recurrence, epidemic prevention staff will quickly find the close contacts using new technologies like big data analysis before the spread in the golden response time (within 24 hours after each outbreak). The aim is to find and control potential infected individuals in advance and try to end the outbreak within one or two maximum incubation periods (Figure 2). The formulation and

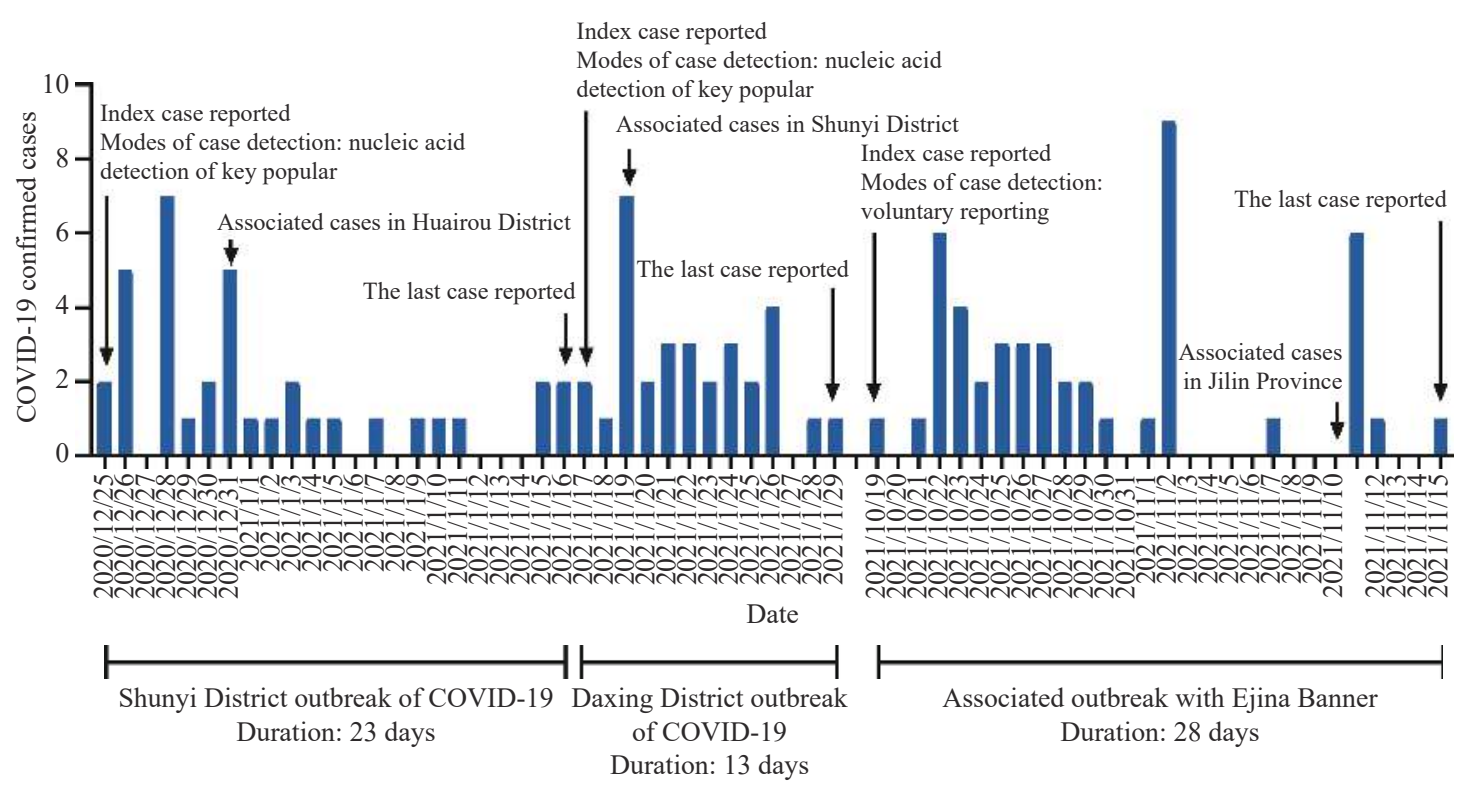

FIGURE 1. Outbreaks of COVID-19 in Beijing from December 2020 to December 2021.

Abbreviation: COVID-19=coronavirus disease 2019. 


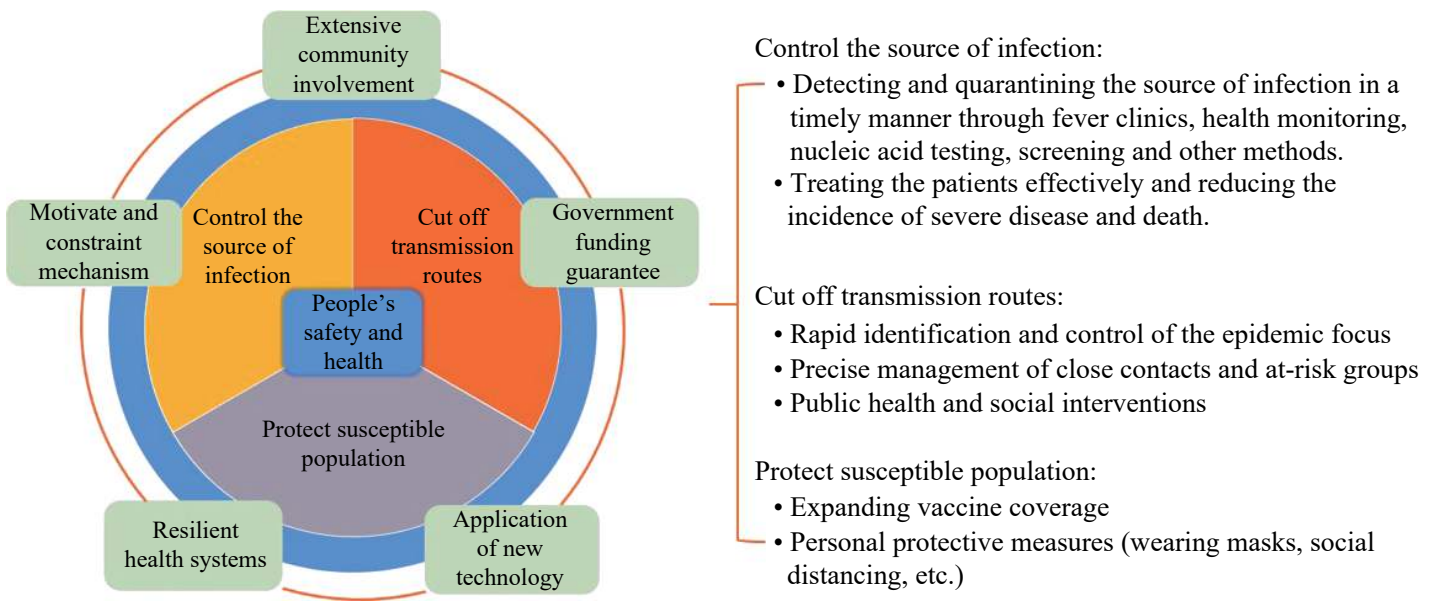

FIGURE 2. The theoretical framework of dynamic COVID-zero strategy.

implementation of this strategy requires extensive community involvement, government funding guarantees, application of new technology, motivating mechanisms, constraint mechanisms, and a resilient health system. Moreover, the scientific suggestions from multidisciplinary experts (for example, public health, clinical medicine, big data analysis, sociology, economics, management, and informatics discipline, etc.) are adopted in a timely manner to support decision making.

With the rapid development of molecular biology technology and the wide use of big data analysis, nucleic acid screening can quickly find the source of infection hidden in the population (5). Strict quarantine and management measures can be subsequently implemented. Big data technology can quickly identify close contacts and risk groups, helping to implement precise prevention and control measures. Compared with severe acute respiratory syndromes (SARS) in 2003, the resilience of China's health system has been improved, and new technologies such as nucleic acid testing and big data analysis have effectively ensured the implementation of the "Dynamic COVID-zero" strategy.

"Dynamic COVID-zero" strategy sums up China's experience in dealing with Delta, Omicron, and other variants, which has advantages in reducing infection. In general, governments adopt country-specific prevention and control strategies based on their COVID-19 situation, health resources, response capacity and final goals (G). No matter what kind of strategy a country takes, concerted and sustained efforts are needed to end the COVID-19 pandemic globally, especially for curbing the rapid spread of the Omicron variant.

Conflicts of interest: No conflicts of interest.
Funding: National Key Research and Development Program of China (2021ZD0114101; 2021ZD0114104; 2021ZD0114105); National Natural Science Foundation of China (72122001; 71934002).

\section{doi: $10.46234 / \mathrm{ccdcw} 2022.015$}

\# Corresponding authors: Min Liu, liumin@bjmu.edu.cn; Wannian Liang, liangwn@tsinghua.edu.cn.

${ }^{1}$ School of Public Health, Peking University, Beijing, China; ${ }^{2}$ Vanke School of Public Health, Tsinghua University, Beijing, China; ${ }^{3}$ Institute for Healthy China, Tsinghua University, Beijing, China.

Submitted: January 19, 2022; Accepted: January 25, 2022

\section{REFERENCES}

1. Haldane V, De Foo C, Abdalla SM, Jung AS, Tan M, Wu SS, et al. Health systems resilience in managing the COVID-19 pandemic: lessons from 28 countries. Nat Med 2021;27(6):964 - 80. http://dx.doi.org/10. 1038/s41591-021-01381-y.

2. Liang WN, Yao JH, Wu J, Liu X, Liu J, Zhou L, et al. Experience and thinking on the normalization stage of prevention and control of COVID-19 in China. Natl Med J China 2021;101(10):695 - 9. http:// dx.doi.org/10.3760/cma.j.cn112137-20210104-00008. (In Chinese).

3. Zhou L, Nie K, Zhao HT, Zhao X, Ye BX, Wang J, et al. Eleven COVID-19 outbreaks with local transmissions caused by the imported SARS-CoV-2 delta VOC - China, July-August, 2021. China CDC Wkly 2021;3(41):863 - 8. http://dx.doi.org/10.46234/ccdcw2021.213.

4. Li ZJ, Chen QL, Feng LZ, Rodewald L, Xia YY, Yu HL, et al. Active case finding with case management: the key to tackling the COVID-19 pandemic. Lancet 2020;396(10243):63 - 70. http://dx.doi.org/10.1016/ S0140-6736(20)31278-2.

5. Ma QY, Liu J, Liu Q, Kang LY, Liu RQ, Jing WZ, et al. Global percentage of asymptomatic SARS-CoV-2 infections among the tested population and individuals with confirmed COVID-19 diagnosis: a systematic review and meta-analysis. JAMA Netw Open 2021;4(12): e2137257. http://dx.doi.org/10.1001/jamanetworkopen.2021.37257.

6. Zhu JM, Yan WX, Zhu L, Liu J. COVID-19 pandemic in BRICS countries and its association with socio-economic and demographic characteristics, health vulnerability, resources, and policy response. Infect Dis Poverty 2021;10(1):97. http://dx.doi.org/10.1186/s40249-02100881-w. 